\title{
DESIGN OF of A PREVENTION SYSTEM OF FRONTAL CAR CRASHES FOR "SACH" CARS
}

Rissetto, Miguel A.; Campos, Juan E.; Bertini Edgardo R.; Prat, Miguel A.; Oris, Ramón; Rodríguez, Gustavo A.

Facultad Regional Avellaneda, Universidad Tecnológica Nacional.

Ramón Franco 5050, (1874), Villa Domínico, Buenos Aires, Argentina.miguelrissetto@gmail.com mrissetto@fra.utn.edu.ar

Facultad Regional Tucumán, Universidad Tecnológica Nacional. Bernardino Rivadavia 1050, (4000), San

Miguel de Tucumán, Tucumán, Argentina, mpratutn@gmail.com

\begin{abstract}
According to the World Health Organization, the number of traffic accidents worldwide reaches 1.25 million people involved in them. This is one of the main causes of death of people between 15 and 29 years old. Most of these deaths are in emergent countries where the rapid economic development has occurred together with an increased use of vehicles. Apart from being a public health issue, the injuries caused by these accidents are an economic-like problem especially in low and medium income countries where their GDP shows about a $3 \%$ economical decrease. Argentine has one of the highest mortality index in the world. In 2017 there were 7.213 deaths (provisional figure obtained up to January 11, 2018). This shows a daily rate of 20 deaths and a month rate of 600 deaths. These indexes raise the need of solving this problem including about 120.000 injured people which occurred annually and the significant material losses. This project comes out with the design of a device to place in old and new cars in order to prevent frontal collisions in Argentine roads, especially in roads with only two lines. This system will first join different types of cameras and sensors operated with a software that will alert the driver in advance in various ways, for example, when a vehicle is coming frontally and the overtake is not possible. The devise could use other technologies such as chips (RID types or similar ones), GPS, laser lights and others considered appropriate to make the device more complete, effective and economically accessible.
\end{abstract}

Key words: anti-collision-car-prevention

\section{INTRODUCTION}

Even though Argentina has a high index of road accident mortality, it is above the rates of America. Argentine has 14 deaths per 1000 inhabitants. It is in the 11 position of the 31 countries analyzed in the World Health Organization report for America. (WHO, 2015). According to the road accidents worldly, statistics show the following data:

1. According to the type of accident, $39 \%$ of the victims drive motorcycles; $32 \%$ are car drivers or people accompanying them and $22 \%$ are pedestrians.

2. According to the victims' age, $56 \%$ are under 35 years old.

3. According to the accident date, $44 \%$ of the victims occurs at weekends.

4. According to the involved car quantity, $33 \%$ of them are caused by only one car.

The present trend shows a $65 \%$ increase in the accident rate which will make the road accidents the fifth mortality cause in 2013 [1]. In 2011, this situation made the United Nations implement a global road safety program called "Decade of action for Road Safety 2011-2020" [2]. The aim of it is to stop accident rates and the main objective is to promote the use of 
technologies that help to avoid or at least to decrease the number of accidents. Regarding these situations, the Government of Sweden is taking the "Zero Vision Initiative" program through which the country is reducing road and street mortality significatively. Zero Vision is the Swedish approach of the road safety concept that can be summarize like this: no loss of lives is acceptable (zero deaths in road accidents). Likewise, this initiative incorporates human errors as part of the calculus proposing four research dimensions to find solutions:

1. Vehicle technology: a number of new systems can decrease drastically deaths related to traffic that causes severe injuries. Technological solutions for cars include from automatic braking systems and Alco locks to airbags technology and so on.

2. Infrastructure: the aim is to build roads and infrastructure that satisfy the environment and capacity challenges without compromising traffic safety. This is not too complicated as it seems to be and it does not need to be more expensive than the traditional way of building roads. Conversely, this will reduce the overall cost for society over time.

3. Traffic and surveillance control: this solution involves systems to monitor the traffic flow and the weather conditions or other actions that are qualified as intelligent traffic systems. Gathering data, measuring results and constantly evaluating the traffic data are crucial. They could be systems to manage weather information, maintenance of traffic flow and much more.

4. Education and training: public services and education are vital in order to implement effective road safety solutions. The approach goes from the driver training to planning services at local and national level. A holistic focus implies to collect knowledge and take out the most effective methods taking into account costs as well as safety. (Available at: http://www.visionzeroinitiative.com).

This project will mainly focus in point 1 mentioned above trying to find the available technology for cars and a solution to the problem without treated in this work disregarding all the ideas summarized in the four points.

\subsection{Car technology}

Even though four research dimensions were mentioned before, the present work is mainly focused on this aspect (car technology). Different devices or car detection systems to avoid accidents will be detailed as stated below starting with the more traditional ones:

a) One of the most basic safety systems is lightening. It has been evolving and allowing increasingly visibility and thus reducing the number of accidents during the night.

b) The ABS (Anti-lock Braking System) and the ESP (Electronic Stability Program) are active safety systems for driving. They are not new. But the protagonists now are the environment detection systems which are still optional equipment. They can help driving be more comfortable and safer being able to avoid and correct slipping and human errors.

The most common errors that drivers make and the existing technology are the following:

a) Sleep detection or lack of attention while driving: this consists of an electronic device with a sensor in the steering wheel that counts the times per minute the driver makes small corrections while driving so the system gives an alert. 


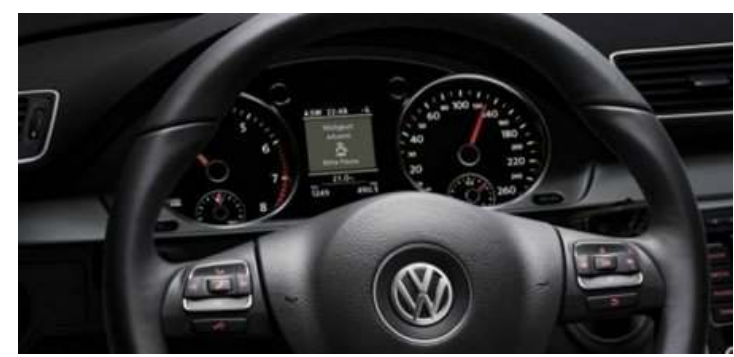

b) Unintentional detection of lane change: it is also known as lane maintenance assist. This system can function as a microprocessor which is permanently watchful that the vehicle path maintains itself between left and right lines of a road.
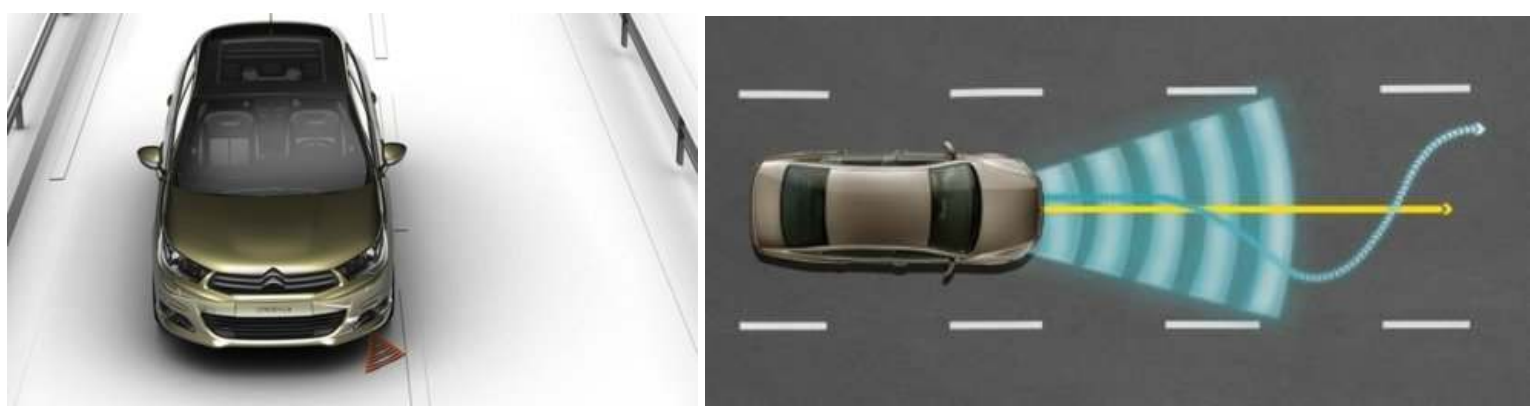

c) Warning of a dead angle (blind side) in the rearview mirror: the dead angle is a small visual field area backwards. It is very near the car that the rearview is not able to reflect. In some vehicles a system warns about the blind side through a visual sign on the mirror or on the frame it is mounted. The system has radars (i.e., they emit radio waves) placed in the corners of the rear bumper or on the car side or sometimes on the rearview mirror itself. Audi A6, for example, has this equipment as an optional one.

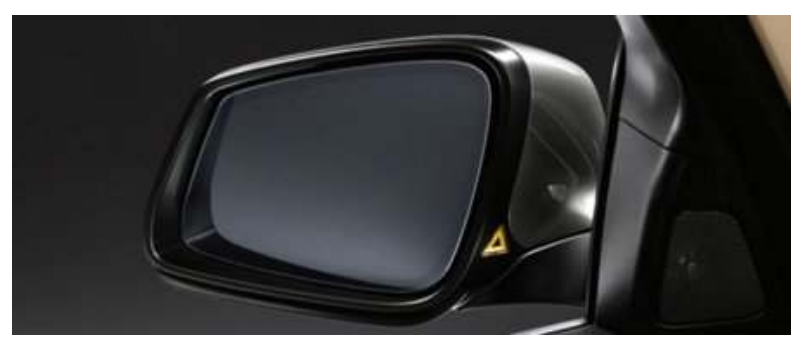

d) Recognition of speed and overtaking signals: a high definition camera with wide-angle lens is placed at the top of the windscreens centered in front of the rearview mirror. It watches out the road sides and recognizes the signals of maximum speed, of overtaking, of end of maximum speed and of prohibited parking. The camera is also used to recognize the lane lines. Opel Astra has incorporated this system. 


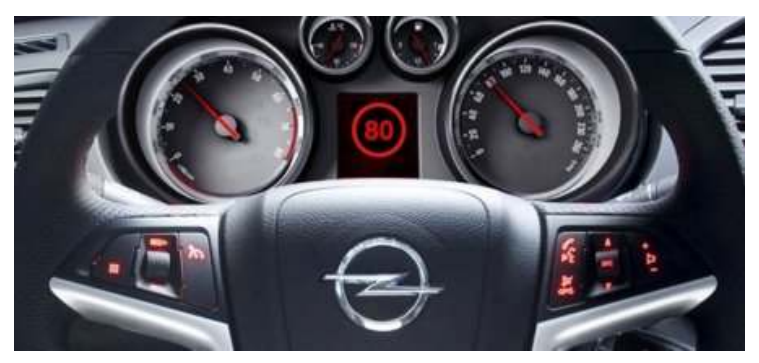

e) Warning of opposite direction: this system is not common. It is found in high-end cars such as Mercedes Benz and BMW. It has a GPS to identify if the driver is about to go to the opposite direction. It tells him/her about it with a visual and sound alarm in the navigator screen. In addition to the alarm for the driver, it sends a warning to the traffic control central and to the cars nearby within a range of $600 \mathrm{~m}$. which in turn has a communication system. It can be also combined with the signal recognition camera.

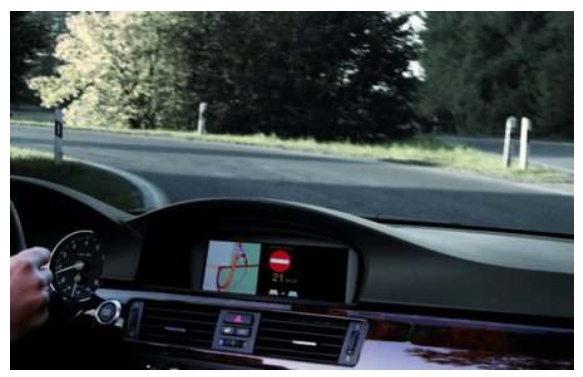

f) Adaptive speed control (with a safety distance system): this system of speed control is increasingly being used including the utilitary cars. It helps to have a more comfortable driving and not to be worried about the speedometer with which the maximum speed would not be exceeded and so avoid a fine. It is very simple. If the gas pedal is electronic, a small processor is in charge of maintaining the programmed speed. A development of this system is called adaptive speed. It keeps steadily the distance of optimum safety if a vehicle is situated in front of it and the driver does not have to worry about the necessary safe distance. A radar is placed on the bumper or front grill. It measures the distance between a car and another one while they are on the road. According to the driving speed, a microprocessor calculates the safety distance between the two cars and it compares them with the existing one. If there is not enough distance, the systems acts over the gas pedal and slows down to keep distance. If it is necessary, it can also function on the brake to decrease speed even more in order to get the needed distance faster. This system is increasingly available as an optional equipment in standard cars and even in hybrid ones. If the safety distance increases and if it is possible, the car will speed up to get back to the programmed speed. The more advanced systems take advantage of the ABS sensors and stability control to recognize the adherence to the road surface and know, for example, if it is wet and thus know that the safety distance must be even higher and adapt automatically to this conditions. 

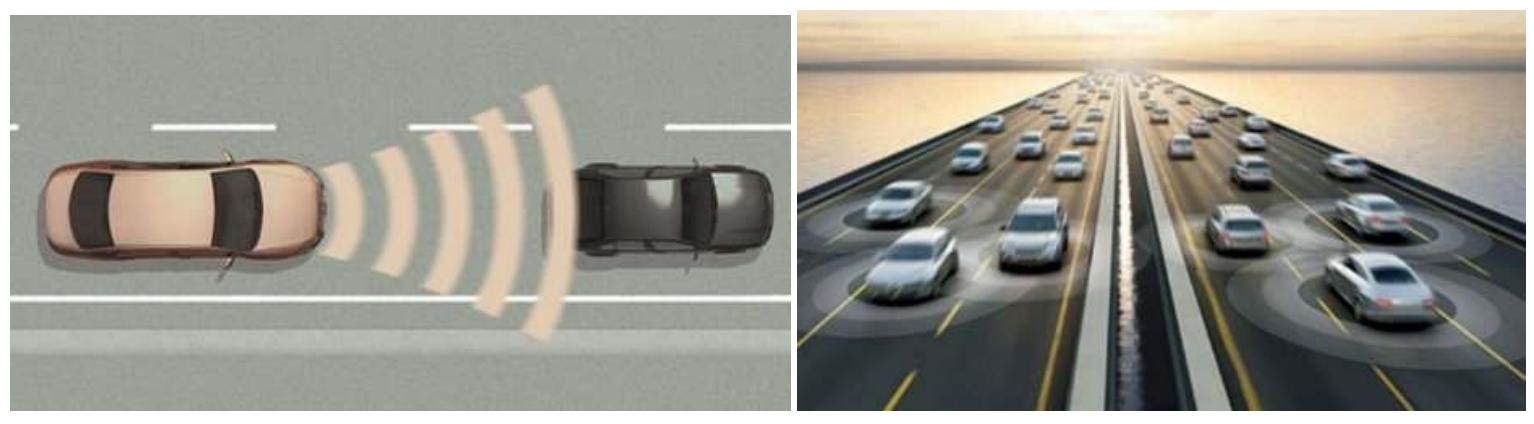

g) Recognition of objects: this system pretends to avoid specially running over pedestrians and cyclists. It is similar to the recognition system of pedestrians at night. The system ContiGuard goes beyond this but it is still experimental. Two high resolution cameras placed on the top of the windshield wipers allow to have a stereographic vision and they are connected to the system microprocessor. It can distinguish among pedestrians, cyclists, cars and other objects. It can measure the distance to them, predict its trajectory and determine if there is risk of an accident in order to take preventive actions to avoid the accident or, at least, to lessen the results. It will warn the driver about these situations and even act on the brakes automatically. Another system that is being tested in Germany called Amullet Car2X works through radio waves. Pedestrians and cyclists should carry a small transponder which is very similar to the RFID system. It does not need electricity if it is of passive type. In this case, the car must carry a transmitter-receiver which captures the response signal of the transponders. This system is good because a pedestrian could be hidden, for example, a child behind a parked car so the microprocessor will know that he/she is there and warn the driver about it.
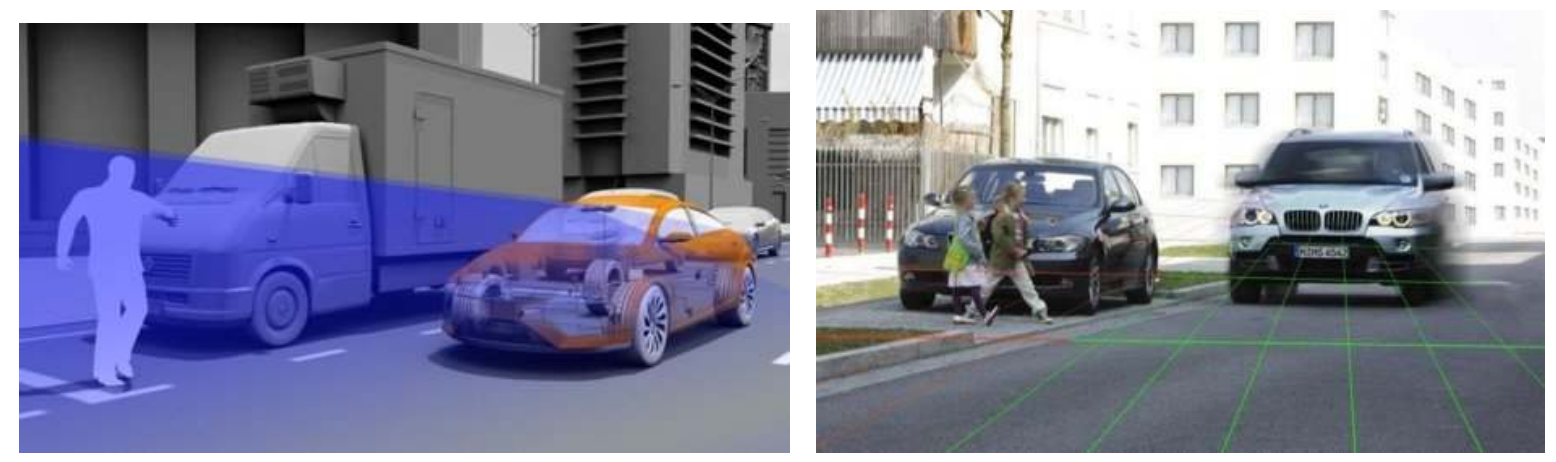

h) Anti-collision system (automatic braking): the electronic "brain" of the car can knows if there are obstacles (pedestrians or another vehicle) in front of it with cameras or radars. So it is determined that there is a real risk of an accident or a collision. The automatic braking systems until the car stops usually function at low speeds (below 40 or $50 \mathrm{~km} / \mathrm{h}$ in the city or traffic jams and doing so, they avoid clueing). Some anti-collision systems only warn the driver and prepare the braking system so that it function with the maximum strength while other systems of this type also brake the vehicle themselves. Volvo (city safety system) and many other brands have this kind of systems. Another experimental system is that of bend anticipation as, for example, the Foresight Transmission Control of BMW. It recognizes the moment the car is arriving to a bend anticipating the possible danger and 
estimates the optimum speed. If the speed is not the appropriate, it automatically brakes to reduce speed apart from decreasing one gear in the gearbox.

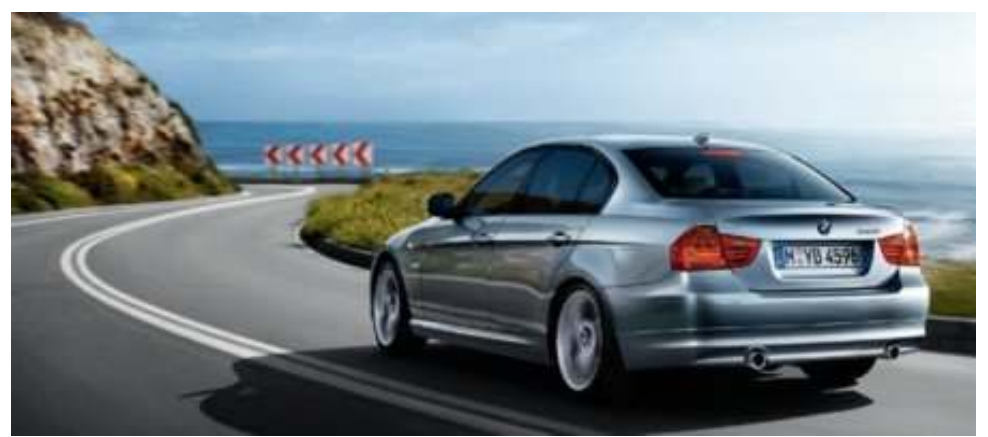

i) Cameras installed for the driver to see what he/she cannot see: this is an optional equipment in mid-range cars such as the Toyota Auris HSD. The gear vision camera is placed above the car license plate. It focuses back and the images are seen in the color screen of the navigation system of the central console. It shows small objects, for example, children who can hidden below the view of the rear window or out of the vision of external rear-view mirror. BMW has already designed this system with the side view cameras placed on the corners of the front bumper which allows the driver to see to the left and right in the road crossings where he/she does not have enough visibility. (In some situations, the vision is a meter and a half or two to the back of the bumper.

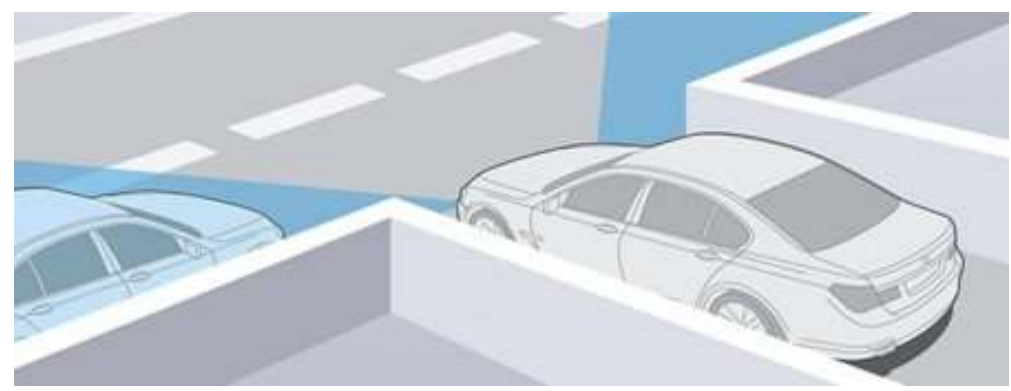

j) Communication systems between cars to give an alert or warn: an example of this type of system is the one that Mercedes Car-to-X has. It is based on the exchange of information between vehicles as well as between vehicles and traffic infrastructure. It has the advantage that it is possible to get information out of the driver's field of vision which does not happen with conventional sensors. This information provides the base several new assistance functions that complement the existing systems. Therefore, the vehicle "sees" the risks before the driver perceives them and it warns him/her and other users on the road or street about the position the car has at real time. When the car receives a risk warning, the Car-to-X system compares the position of the vehicle in danger. As soon as the car is approaching to a dangerous point, the system warns the driver by an audible and visual way. This allows the driver to be prepared to the situation, adjust his driving and avoid accidents. These traffic networks are not only safe but also more efficient because when accidents are prevented, traffic lows more free and efficient. The Car-to-X communication can contribute to an efficient mobility as well through a network connection with the traffic infrastructure such as traffic lights. The system can even make the other safety systems be 
alert and functions automatically even before the driver reacts to the alert message and reacts.

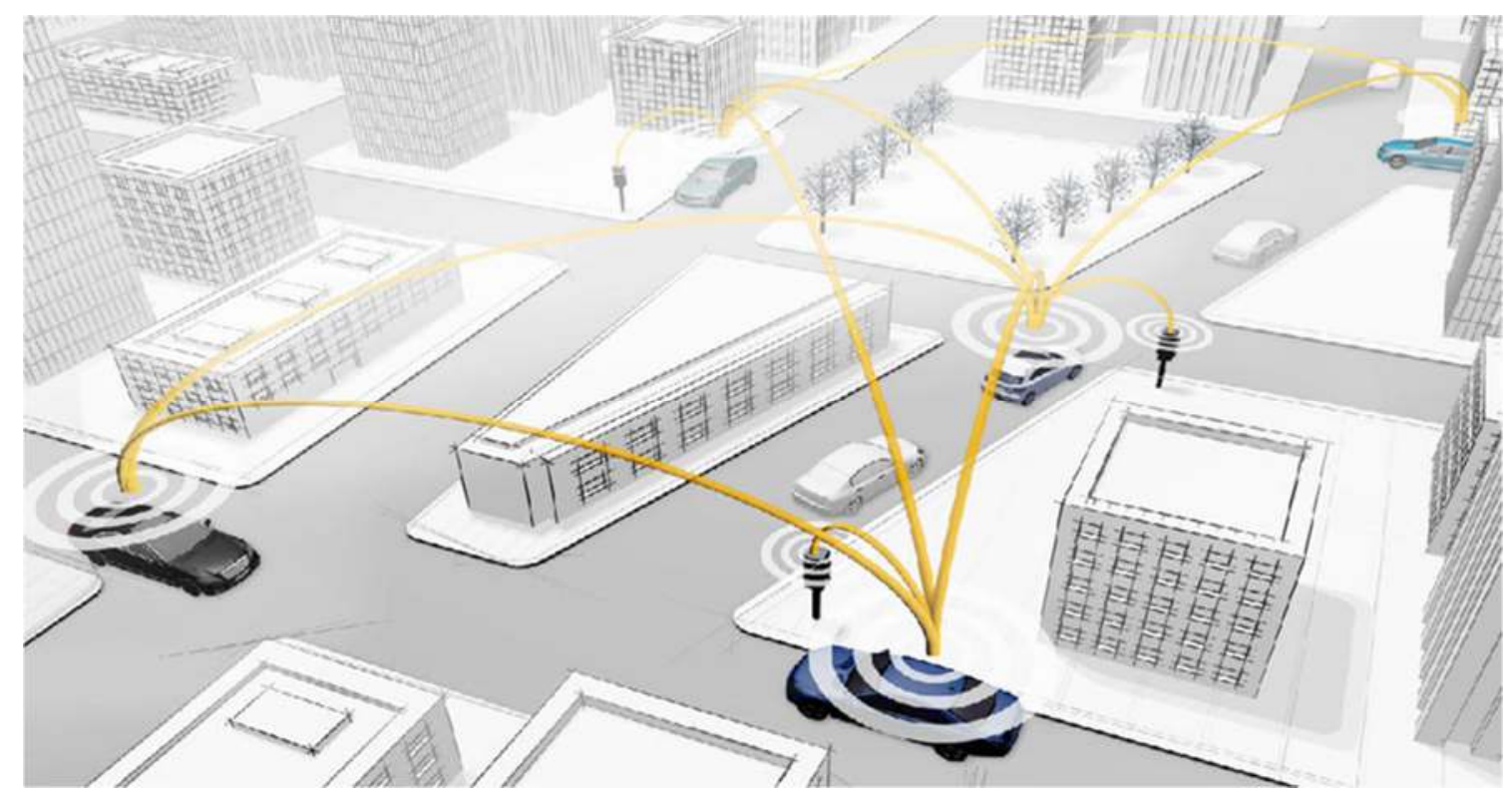

As it can be seen, there are many systems very related to each other. Unfortunately, we will have to wait until its price lowers and be incorporated to more economic vehicle brands and models. Safety should always be a standard equipment.

\section{2-OBJECTIVES}

The present work is the first advance of a Research Project that has the same name and it is taken place at the UTN. Their main objectives are as follows:

\subsection{Main objective}

--Diminish significantly deaths caused by car accidents in roads and cities in the Argentine Republic.

\subsection{Secondary objectives}

-Design an economically accessible product/system so that it can be installed in every vehicle either in a private way or through a national sate initiative in new or old cars.

-Avoid basically frontal collisions, mainly with dead victims, that happen principally in roads with only one side lane.

-Avoid accidents that occur when animals are free in roads and paths.

-Diminish the collisions caused by distraction, lack of calculus, lack of vision or sudden braking with the vehicles circulating frontally in the same direction.

-Prevent the car to hit pedestrians, cyclists and cars in city cross streets, abrupt exits from the back of parking cars or accidents in bad weather conditions such as fog, poor lighting, night and so on.

-Evaluate the social and economic impact caused by the implementation of this system. 


\section{3-DESIGN PROPOSALS}

After the researches made of the existing systems and devices worldwide and taking into account that there is little of these equipment implemented in Argentine, it was decided to go forward with those that are considered the most appropriate alternatives. First, because of the technologies available in the country and then because of the possibility to get this equipment at low costs and so most of the vehicles can have it. The latter is essential in the sense that the social impact of this project would represent a real fast definite possible solution for a country that has one of the worst positions in the world regarding accidents and deaths caused by vehicles.

Regarding this situation, the most appropriate studied variables are shown as follows:

\subsection{Wide-angle focus and long distance camera placed on the rear-view mirror of the driver's side and focused towards the front:}

This position will allow not to be necessary to lean out to look if it is possible to pass a car. The camera will have:

-Infrared viewer for day and night in roads without lighting with detector and recognition of images to differentiate trucks, cars, bicycles, dangerous animals (cows, horses, deer, sheep, dogs, fowls and so on), signals, etc.

-A screen either the one provided in the car or placing a new one. It can be connected to the GPS or a smartphone with wire or wireless.

-Sound: everything that the screen shows will be announced with voice and also with sound alarms announcing danger or risks.

-Lights for danger warning.

-Software: a design of a specific software is thought to process the speed data of the vehicle. The operation of this software will be as follows:

a) The detected images are compared with a database previously charged to be used. Distance, movement as well as heat will be analyzed through sensors or images.

b) The type of object is analyzed. If it is at rest and, for example, it is a signal, it does not announce the danger. But if it is an animal, it does warn about the danger (temperature sensor). It also warns about an object in movement (car, person or animal). Considering these situations, speed is processed and it detects if the object goes on the same direction or on the contrary one. (it can be connected to the GPS of the car). If a car is going on the opposite direction, the system gives a report about distance and analyzes if it is possible to overtake. To do so, the system charges information about the car itself in tests or by data provided by the manufacturer. The characteristics of the driver are also charged (driving style, age, seeing, reflexes, etc.). If the system concludes that the overtake is not possible, it sends a warning through an image, voice or sound alarm. If the overtake is possible, the device reports about the distance to the object and the suggested gear, for example $3^{\text {rd }}$, to use at the proper time. The software could also recognize the roadside and the demarcation lines. The camera shows and detects if the preceding bend is to the left or right in order to inform the driver. It also shows if the car goes out of the road.

\subsection{Alternatives to include eventually in the device}


-Adding a camera on the bumper to detect if a car comes from a street or if a person goes out from the back of a truck or similar situations.

-Installing a laser light below the front bumper. That light is projected on the road anticipating the car movement. This line will permit that the car driver who is coming on the opposite direction see the light much before the car reaches him/her. This light must be seen during the day and night. This system can also show a hologram-like image.

-The camera mentioned in 3.1. can be supplemented or replaced by a measurer of laser distance or a sound that informs about distance-proximity to the object that comes in the opposite direction or simply detects objects at less safe distance of the overtake. Thus, it only informs if the car can pass because if it does not detect anything it means that the object is far or there is nothing. This distance can be programmed based on the car characteristics (reaction or acceleration) and on the driver's characteristics. This system would be cheaper and simpler. The detectors can be:

a) One device focuses the other roadside. It is situated on the driver's side mirror to avoid frontal collisions.

b) Another device focuses the road ahead. It is situated on the grill to advice that a car is approaching dangerously to an object that can be at rest and cannot be seen such as broken trucks or cars stopped on the side-road or that can be driving at slow speed because of fog.

\subsection{Complementary alternatives that can favor, enlarge and enhance the success of the proposed device:}

-Installation of a chip or RFID sensor which very cheap and produces radio waves in every car, people, bicycles, etc. so that it can give radar-type information about proximity. It can be analyzed by the mentioned software and give complementary data for a better safety. The signal of the chip or RFID can be replaced or supplemented by a mobile phone, radio, Wi-Fi or Bluetooth detecting different waves contributing with more exact data to the system.

-System " $a$ " can be replaced by a radar-sonar system with the same aim.

-These systems can be supplemented with systems placed in the roads and sends the necessary information to the car having the device (radio, GPS, etc.) through solar energy antennas. These posts can be replaced by drones reporting online about the road state.

-Another additional system that can be employed is a RFID sensor or a chip or another detector (for example, placed on the license plate) and provided by the respective City Councils. It will be noticed by a tracking system each car has and will detect the proximity of the vehicles whether they go on the same direction or on the opposite one. This signal will appear in the GPS screen or it can only be a sound signal that will warn about the presence of a car, for example, by the GPS or radio reporting the distance of the car. The possibility or impossibility of passing a car on the road or any another risky situation will be recommended through a software that processes the speed of nearby vehicles which compares it with the car's speed itself.

-The most simple alternative, unique or combined, that can be applied with the other proposed systems, i.e., warning the cars coming on the contrary lane that my car is approaching. It would be a more specific and combined system than the lights on during day and night. The proposal is then a laser placed in the front part of the car projecting a light beam over the road 
$1000 \mathrm{~m}$. away forward. This distance can be verified and maybe it can be adjusted automatically according to the car speed. The aim of this beam is to warn -anticipate- that the cars coming on the contrary way without the need of "leaning out". So, when the driver sees the light, he/she knows that a car is approaching at $1000 \mathrm{~m}$. and thus, avoiding the dangerous maneuvering of leaning out or to make bad calculi of distance.

The speed of the technological advances and the decrease of costs compel us to think about other possibilities of solutions to the mentioned ones.

\section{4) CONCLUSIONS}

There is not a product or system in the market that accomplishes the requirements of this investigation either because of their costs or technologies to which general public can access easily. With these carried out researches and analysis, it is possible to conclude that the relevant technology are available to go forward to the design of a product that decreases the frontal car crashes on the roads in an economical and efficient way. Likewise, being a system constituted by an equipment that will include different sensors, cameras, laser, other equipment and also a specific assistance software for the driver, it will be possible to give answers to the objectives this research proposes. Generating an impact by far positive in the community will decrease drastically the accidents and deaths in Argentina.

\section{5) REFERENCES}

[1] Motorpasión Futuro | Continental ContiGuard: prevención de accidentes mediante la detección de objetos, AMULETT CAR-2-X, sistema de radio para evitar atropellos, Toyota Prius, Top 10 de los coches innovadores, BMW crea una tecnología capaz de predecir las curvas, Toyota Auris HSD, Ford CoCarX: el coche que se comunica evita accidentes, Audi A6 hybrid, Volvo intenta evitar el atropello de animales.

[2]Motorpasión | Citroën C4, Volkswagen Passat

[3]Xataka | Sistemas de iluminación avanzados en coches / Xataka en Instagram

1. World Health Organization, WHO: Global status report on road safety 2009. URL:http://www.who.int/violence_injury_prevention/road_safety_status/2009

2. WHO: Decade of action for road safety 2011-2020: Global Launch. URL

http://www.who.int/roadafety/publication/decade launch

\section{ACKNOWLEDGMENTS}

The authors of this paper would like to thank Facultad Regional Tucuman, Universidad Tecnologica Nacional for their support to go ahead with this work and thus having the opportunity of reduce accidents in Argentine roads.

Among the numerous people that have helped us, we wish to thank Universidad Tecnologica Nacional and Direccion de Accion Social (DASUTEN) for their initiatives and projects related to the prevention of road accidents.

Finally, we would like to thank Professor Silvina Alicia Bascary for the translation of this work from Spanish to English. 